\title{
CDISC SDTM Location of Most Severe Stenosis Within a Graft Terminology
}

National Cancer Institute

\section{Source}

National Cancer Institute. CDISC SDTM Location of Most Severe Stenosis Within a Graft

Terminology. NCl Thesaurus. Code C101851.

Terminology associated with the location of the most severe stenosis within a graft codelist of the Clinical Data Interchange Standards Consortium (CDISC) Study Data Tabulation Model (SDT M). 